\title{
Structural Health Monitoring using Shaped Sensors
}

\author{
Michael I. Friswell* and Sondipon Adhikari ${ }^{\dagger}$ \\ Swansea University, UK
}

\begin{abstract}
This paper is concerned with distributed sensors to measure the response of beam and plate structures. The design of modal sensors for beam structures is well established. The design for plate structures with constant thickness sensors may be achieved by optimizing the shape of the sensor boundary, or by optimizing the effectiveness of the electrode. Most applications consider vibration control, but this paper is concerned with structural health monitoring, where the sensor output is made sensitive to changes in key stiffness parameters, for example in joints. The procedure is based on finite element models of the structures, and thus distributed transducers may be designed for arbitrary beam and plate structures. A simulated beam excited by a rotating machine is used to demonstrate the approach.
\end{abstract}

\section{Introduction}

The idea of using modal sensors and actuators for beam and plate type structures has been a subject of intense interest for many years. Using modal sensors in active control reduces problems of spillover, where high frequency unmodeled modes affect the stability of the closed loop system. The sensors and actuators may be discrete or distributed, and are usually manufactured using piezoelectric material, such as polyvinylidene fluoride (PVDF) film. For example, a modal sensor for a beam type structure may be obtained by varying the sensor width along the length of the beam. If the sensor covers the whole beam the shape of the sensor may be derived using the mode shape orthogonality property. ${ }^{1,2}$ Modal sensors may be designed that cover only part of the beam ${ }^{3}$ or are segmented sensors sensitive to multiple modes. The effect of geometric tolerances during manufacture on the quality of the sensors may be determined. ${ }^{3}$ For beam structures the width of the sensor may be parameterized using the finite element method and the underlying shape functions used to approximate the transducer shape. ${ }^{4}$

For two-dimensional structures, the approach used for beams may be implemented by varying the thickness of the PVDF, although this is very difficult to achieve in practice. Sun et al. ${ }^{5}$ replaced an actuator layer with variable thickness by many small segments of uniform thickness. Kim et al. ${ }^{6,7}$ developed two design methods for distributed modal transducers for composite plates, the first using multi-layered PVDF films with optimized electrode pattern, lamination angle, and poling direction, and the second using PVDF film segments and an interface circuit. Preumont et al. ${ }^{8}$ introduced the porous electrode concept, which allows the gains to be introduced by changing the local effectiveness of the electrodes. The alternative is to design a distributed modal transducer by optimizing the continuous boundary shape of a constant thickness PVDF film by assuming a smooth boundary. ${ }^{9-11}$

This paper uses the design methods for modal sensors, but extends this approach to the structural health monitoring application.

The identification of the location and severity of cracks, loose bolts and other types of damage in structures using vibration data has received considerable attention. ${ }^{12}$ Most of the approaches use the modal data of a structure before damage occurs as baseline data, and all subsequent tests are compared to it. ${ }^{13-16}$ Any deviation in the modal properties from this baseline data is used to estimate model parameters related to the damage severity and location. The advantage of using this baseline data is that some allowance is made for modeling errors. However changes in the structure not due to damage, for example due to environmental effects, will be difficult to distinguish from changes due to damage. ${ }^{17,18}$ The approach adopted in this paper

${ }^{*}$ Professor of Aerospace Structures, School of Engineering, Swansea SA2 8PP.
${ }^{\dagger}$ Professor of Aerospace Engineering, School of Engineering, Swansea SA2 8PP. 
is to use shaped transducers to reduce the sensitivity of the sensor output to the unmodeled parameter changes and environmental effects. For structural health monitoring this means that the response can be made sensitive to particular regions of interest, so that, for example, the sensor may be used to monitor the health of a single joint. The method is an extension of the selective sensitivity technique which was developed to design excitations that produce strong sensitivities to a subset of the parameters whilst causing the sensitivities to other parameters to vanish. This concept is modified to choose the shaped sensor, although the spatial and spectral properties of the expected in-service excitation are still an important aspect of the design.

The finite element model of the structure is of the form

$$
\mathbf{M} \ddot{\mathbf{q}}+\mathbf{D} \dot{\mathbf{q}}+\mathbf{K q}=\mathbf{B u}, \quad \mathbf{y}=\mathbf{C q}
$$

where $\mathbf{M}, \mathbf{D}$ and $\mathbf{K}$ are the mass, damping and stiffness matrices based on the degrees of freedom, $\mathbf{q}$. The inputs to the structure, $\mathbf{u}$, are applied via a matrix, $\mathbf{B}$, which determines the location and gain of the actuators (or the actuator shape for distributed actuators). Similarly the outputs, $\mathbf{y}$, are obtained via the output matrix $\mathbf{C}$ which is determined by the sensor shape. For parameter estimation and health monitoring applications the mass, damping and stiffness matrices depend on a set of physical parameters, $\theta$.

\section{Defining Shaped Sensors}

For beam structures whose response is predominantly in bending in a single plane, the transducer may be shaped by varying the width of the PVDF material. For beam and plate structures either the thickness ${ }^{5-7}$ or the electrode effectiveness ${ }^{8}$ (which may be considered equivalent to thickness) may be changed. In all these cases the width or thickness is assumed to be a continuous function. However this function needs to be parameterized to enable the optimization of the sensor. Using the shape functions of the underlying finite element model is a convenient approach to approximate the width or thickness of the piezoelectric material. ${ }^{4}$ In this way modal transducers may be designed for arbitrary beam or plate type structures, and the sensor width or thickness (and often its slope also) will be continuous across the element boundaries. Furthermore modal transducers that only cover part of a structure may be designed. Most of the development will concern sensors, although actuators may be dealt with in a similar way.

For a plate element, the output (voltage or charge) from the part of the sensor covering element number $e$ is 5

$$
y_{e}(t)=K_{s} \iint f_{e}(\xi, \eta)\left(\frac{\partial^{2} w_{e}(\xi, \eta, t)}{\partial \xi^{2}}+\frac{\partial^{2} w_{e}(\xi, \eta, t)}{\partial \eta^{2}}\right) \mathrm{d} \xi \mathrm{d} \eta
$$

where $f_{e}(\xi, \eta)$ defines the effectiveness of the sensor at location $(\xi, \eta)$ which incorporates the sensor thickness (or possibly distance from the plate neutral plane), the piezoelectric coefficient and also the polarization direction. Here a Cartesian coordinate system is used and the integration is performed over the element, although other coordinate systems could be used. $w_{e}$ is the plate deflection within the element. $K_{s}$ is a constant relating to the piezoelectric properties. For a rectangular plate element, with dimensions $2 a$ and $2 b$ in the $\xi$ and $\eta$ directions respectively, the output would be

$$
y_{e}(t)=K_{s} \int_{-b}^{b} \int_{-a}^{a} f_{e}(\xi, \eta)\left(\frac{\partial^{2} w_{e}(\xi, \eta, t)}{\partial \xi^{2}}+\frac{\partial^{2} w_{e}(\xi, \eta, t)}{\partial \eta^{2}}\right) \mathrm{d} \xi \mathrm{d} \eta .
$$

For a beam element of length $\ell_{e}$ the output is

$$
y_{e}(t)=K_{s} \int_{0}^{\ell_{e}} f_{e}(\xi) \frac{\partial^{2} w_{e}(\xi, t)}{\partial \xi^{2}} \mathrm{~d} \xi
$$

where now the sensor effectiveness $f_{e}(\xi)$ allows for varying width, as well as (possibly) varying electrode effectiveness or thickness. The development will concentrate on sensors for plate structures, although it is clear that the results for beam structures will be a special case.

The objective is to approximate the continuous sensor effectiveness using a discrete set of parameters, based on the finite element shape functions. In finite element analysis the displacement is approximated as

$$
w_{e}(\xi, \eta)=\mathbf{N}(\xi, \eta) \mathbf{q}_{e}
$$


where $\mathbf{N}(\xi, \eta)$ are the shape functions and $\mathbf{q}_{e}$ are the nodal generalized displacements. Suppose that the sensor thickness is also approximated by the shape functions, so that

$$
f_{e}(\xi, \eta)=\mathbf{N}(\xi, \eta) \mathbf{f}_{s e}
$$

where $\mathbf{f}_{s e}$ is a vector of the same length as the nodal displacements, that defines the thickness of the sensor. In this way the continuous sensor thickness is approximated using a finite set of parameters in $\mathbf{f}_{s e}$. Substituting into Eq. (2) gives

$$
y_{e}(t)=\mathbf{f}_{s e}^{\top} \mathbf{C}_{s e} \mathbf{q}_{e}
$$

where

$$
\mathbf{C}_{s e}=K_{s} \iint \mathbf{N}(\xi, \eta)^{\top}\left(\frac{\partial^{2} \mathbf{N}(\xi, \eta)}{\partial \xi^{2}}+\frac{\partial^{2} \mathbf{N}(\xi, \eta)}{\partial \eta^{2}}\right) \mathrm{d} \xi \mathrm{d} \eta .
$$

The total sensor output is obtained by summing the output from all of the elements, thus

$$
y=\sum_{e} y_{e}=\mathbf{f}_{s}^{\top} \mathbf{C}_{s} \mathbf{q}
$$

where $\mathbf{q}$ is the vector of generalized displacements of the full model, and $\mathbf{f}_{s}$ is a vector incorporating the sensor parameter vectors. In this way, the problem of a continuous thickness variation is changed into a discrete optimization problem for $\mathbf{f}_{s}$. The assembly process is exactly analogous to the assembly of mass and stiffness matrices in standard finite element analysis. The only slight difference is the incorporation of boundary conditions. For example a pinned boundary will require that some of the generalized displacements are set to zero, which reduces the number of degrees of freedom. Just because a generalized displacement at a given node is zero does not mean that the corresponding element of the sensor thickness parameters, $\mathbf{f}_{s}$, should be zero, as the sensor thickness may be non-zero at the boundary. In turn this means that $\mathbf{C}_{s}$ may be a rectangular matrix. Comparing Eqs. (1) and (9) it is clear that $\mathbf{C}=\mathbf{f}_{s}^{\mathrm{T}} \mathbf{C}_{s}$.

For a beam element with cubic shape functions, ${ }^{4}$

$$
\mathbf{C}_{s e}=-\frac{K_{s}}{30 \ell_{e}}\left[\begin{array}{cccc}
36 & 33 \ell_{e} & -36 & 3 \ell_{e} \\
3 \ell_{e} & 4 \ell_{e}^{2} & -3 \ell_{e} & -\ell_{e}^{2} \\
-36 & -3 \ell_{e} & 36 & -33 \ell_{e} \\
3 \ell_{e} & -\ell_{e}^{2} & -3 \ell_{e} & 4 \ell_{e}^{2}
\end{array}\right]
$$

For plate elements the matrices are relatively easy to generate, although difficult to express in a closed and compact form. Dawe ${ }^{19}$ described the R1 rectangular element which has four nodes and three degrees of freedom per node. As an example, consider a square element with side $2 a$. Then the element output matrix, $\mathbf{C}_{s e}$, is

$$
\frac{K_{s}}{720}\left[\begin{array}{cccccccccccc}
-600 & -120 a & -120 a & 168 & 48 a & 0 & 264 & -72 a & -72 a & 168 & 0 & 48 a \\
-552 a & -143 a^{2} & -159 a^{2} & -240 a & -79 a^{2} & 111 a^{2} & 24 a & 31 a^{2} & 15 a^{2} & 48 a & 47 a^{2} & -15 a^{2} \\
-552 a & -159 a^{2} & -143 a^{2} & 48 a & -15 a^{2} & 47 a^{2} & 24 a & 15 a^{2} & 31 a^{2} & -240 a & 111 a^{2} & -79 a^{2} \\
168 & 48 a & 0 & -600 & -120 a & 120 a & 168 & 0 & -48 a & 264 & -72 a & 72 a \\
-240 a & -79 a^{2} & -111 a^{2} & -552 a & -143 a^{2} & 159 a^{2} & 48 a & 47 a^{2} & 15 a^{2} & 24 a & 31 a^{2} & -15 a^{2} \\
-48 a & 15 a^{2} & 47 a^{2} & 552 a & 159 a^{2} & -143 a^{2} & 240 a & -111 a^{2} & -79 a^{2} & -24 a & -15 a^{2} & 31 a^{2} \\
264 & 72 a & 72 a & 168 & 0 & -48 a & -600 & 120 a & 120 a & 168 & -48 a & 0 \\
-24 a & 31 a^{2} & 15 a^{2} & -48 a & 47 a^{2} & -15 a^{2} & 552 a & -143 a^{2} & -159 a^{2} & 240 a & -79 a^{2} & 111 a^{2} \\
-24 a & 15 a^{2} & 31 a^{2} & 240 a & 111 a^{2} & -79 a^{2} & 552 a & -159 a^{2} & -143 a^{2} & -48 a & -15 a^{2} & 47 a^{2} \\
168 & 0 & 48 a & 264 & 72 a & -72 a & 168 & -48 a & 0 & -600 & 120 a & -120 a \\
-48 a & 47 a^{2} & 15 a^{2} & -24 a & 31 a^{2} & -15 a^{2} & 240 a & -79 a^{2} & -111 a^{2} & 552 a & -143 a^{2} & 159 a^{2} \\
-240 a & -111 a^{2} & -79 a^{2} & 24 a & -15 a^{2} & 31 a^{2} & 48 a & 15 a^{2} & 47 a^{2} & -552 a & 159 a^{2} & -143 a^{2}
\end{array}\right]
$$




\section{Health Monitoring and Selective Sensitivity}

A significant problem with any inverse problem, such as structural health monitoring, is ill-conditioning of the estimation equations. One major cause of this ill-conditioning is the large number of candidate parameters that may be regarded as uncertain. Applying excitations that produce strong sensitivities to a subset of the parameters whilst causing the sensitivities to other parameters to vanish is one way of reducing the number of parameters to estimate. For structural health monitoring this means that the response can be made sensitive to particular regions of interest, so that, for example, the sensor may be used to monitor the health of a single joint. One approach to local structural health monitoring is to use high frequency impedance measurements, ${ }^{20}$ although using shaped distributed actuators and sensors could also be applied to these impedance techniques.

The method of selective sensitivity considers the response predictions to a relatively large number of excitation forces. In order to provide further explanation, the work of Ben-Haim ${ }^{21-24}$ will be adapted to sensors rather than actuators. The selective sensitivity approach is best derived in the frequency domain. From Eq. (1)

$$
\mathbf{y}(\omega, \theta)=\mathbf{C}\left[-\omega^{2} \mathbf{M}+\mathbf{j} \omega \mathbf{D}+\mathbf{K}\right]^{-1} \mathbf{B u}(\omega)=\mathbf{f}^{\top} \mathbf{C}_{s} \mathbf{H}(\omega, \theta) \mathbf{B u}(\omega)
$$

where $\mathbf{H}(\omega, \theta)=\left[-\omega^{2} \mathbf{M}+\mathbf{j} \omega \mathbf{D}+\mathbf{K}\right]^{-1}$ is the frequency response function (receptance matrix).

At any frequency the sensitivity of the response to parameter $\theta_{j}$ is given by

$$
S_{j}(\mathbf{f}, \omega)=\left\|\frac{\partial y}{\partial \theta_{j}}\right\|^{2} .
$$

Notice that only a single output has been assumed, so that $y$ is a scalar, although the approach is easily extended to multiple outputs. Then

$$
S_{j}(\mathbf{f}, \omega)=\mathbf{f}^{\top} \mathbf{C}_{s} \frac{\partial \mathbf{H}}{\partial \theta_{j}} \mathbf{B} \mathbf{u u}{ }^{\mathrm{H}} \mathbf{B}^{\mathrm{H}} \frac{\partial \mathbf{H}^{\mathrm{H}}}{\partial \theta_{j}} \mathbf{C}_{s}^{\top} \mathbf{f}=\mathbf{f}^{\top} \mathbf{G}_{j}(\omega) \mathbf{f}
$$

where $\mathbf{G}_{j}(\omega)$ may be calculated and the superscript $H$ denotes the conjugate transpose. $\mathbf{G}_{j}(\omega)$ is usually complex, although the imaginary part of the matrix is skew-symmetric so that the sensitivity is real. Thus the imaginary part of $\mathbf{G}_{j}(\omega)$ does not affect the sensitivity and may be neglected. Note that for excitation at a single location, $\mathbf{G}_{j}(\omega)$ has rank 1. The derivative of $\mathbf{H}$ may be calculated as

$$
\frac{\partial \mathbf{H}}{\partial \theta_{j}}=\mathbf{H}\left[-\omega^{2} \frac{\partial \mathbf{M}}{\partial \theta_{j}}+\mathrm{j} \omega \frac{\partial \mathbf{D}}{\partial \theta_{j}}+\frac{\partial \mathbf{K}}{\partial \theta_{j}}\right] \mathbf{H} .
$$

The sensitivity defined in Eq. (14) varies with frequency. For the standard selective sensitivity method the force input is designed and so may be varied with both location and frequency. With a shaped sensor for health monitoring the excitation is likely to be due to either ambient forces or operational conditions. Furthermore, to use a simple threshold to determine damage the output required is a single response quantity. In these cases an integrated sensitivity may be defined as

$$
\hat{S}_{j}(\mathbf{f})=\int_{\omega_{1}}^{\omega_{2}} W(\omega) S_{j}(\mathbf{f}, \omega) \mathrm{d} \omega
$$

for some frequency weighting function $W(\omega)$ (that can be designed along with the sensor shape) and frequency range $\left(\omega_{1}, \omega_{2}\right)$. For a given force input

$$
\hat{S}_{j}(\mathbf{f})=\mathbf{f}^{\top}\left[\int_{\omega_{1}}^{\omega_{2}} W(\omega) \mathbf{G}_{j}(\mathbf{f}, \omega) \mathrm{d} \omega\right] \mathbf{f}=\mathbf{f}^{\top} \hat{\mathbf{G}}_{j} \mathbf{f} .
$$

If the location and spectrum of the force is not known then this makes the design of a selectively sensitive sensor extremely difficult. The rest of the development will consider only a single frequency using $\mathbf{G}_{j}(\omega)$ defined in Eq. (14), although the methods are readily extended to the frequency weighted versions using $\hat{\mathbf{G}}_{j}$ defined in Eq. (17). 
The selective sensitivity problem for parameter $s$ is to design the sensor such that

$$
S_{j}(\mathbf{f})= \begin{cases}\neq 0 & \text { if } j=s \\ =0 & \text { otherwise }\end{cases}
$$

Since the sensitivities are all real and non-negative, the conditions in Eq. (18) may be written as

$$
S_{s}(\mathbf{f})=\mathbf{f}^{\top} \mathbf{G}_{s} \mathbf{f} \neq 0, \quad \sum_{j \neq s} S_{j}(\mathbf{f})=\sum_{j \neq s} \mathbf{f}^{\top} \mathbf{G}_{j} \mathbf{f}=\mathbf{f}^{\top} \tilde{\mathbf{G}}_{s} \mathbf{f}=0
$$

where $\tilde{\mathbf{G}}_{s}=\sum_{j \neq s} \mathbf{G}_{j}$.

Solving the selective sensitivity requirements exactly is often not possible, in the sense that a solution may not exist, or may not be unique. One straight-forward approach is to employ an optimization scheme directly. For example the sensor shape $\mathbf{f}$ sensitive to parameter $\theta_{s}$ is obtained as the vector that minimizes the objective function

$$
J(\mathbf{f})=\left\{\sum_{j \neq s} W_{j} S_{j}(\mathbf{f})\right\} / S_{s}(\mathbf{f}),
$$

where $W_{j}$ are weighting factors for the different parameters. The thickness of the sensor is then normalized to the maximum thickness required.

\section{A. Practical Implementation}

However this simple approach may lead to difficulties if the solution is not unique and hence there are many solutions for perfect sensors that satisfy Eq. (18). If the excitation is at a single degree of freedom then the complex matrix $\mathbf{G}_{j}(\omega)$ will have rank 1, although the real part alone will usually have rank 2 . If there are $p$ parameters, and the response should be insensitive to $p-1$ of them, then the dimension of the space of suitable sensor shape vectors, $\mathbf{f}$, based on Eq. (18), is $n-2 p+2$, where $n$ is the length of the vector $\mathbf{f}$. In this case one has to introduce other regularizing conditions into the optimization. One possibility is to reduce the number of elements that the sensor covers, and subset selection techniques could be used to determine the optimum choice of elements. Another possibility is to minimize the transducer curvature, ${ }^{4}$ which has the advantage to producing sensors that are easier to manufacture. For beam structures, minimizing transducer curvature for a sensor covering the whole beam was shown to be equivalent to minimizing $\mathbf{f}^{\top} \hat{\mathbf{K}} \mathbf{f}$ where $\hat{\mathbf{K}}$ is the stiffness matrix for the free-free beam. This is equivalent to minimizing a strain energy type expression, and motivates the optimization problem for the general system.

The best approach is to enforce the zero sensitivity to unwanted parameters before optimizing any other objectives. Thus a transformation, $\mathbf{T}$, is introduced such that

$$
\mathbf{T}^{\top} \tilde{\mathbf{G}}_{s} \mathbf{T}=\mathbf{0}
$$

This transformation matrix may be found easily using the singular value decomposition of $\tilde{\mathbf{G}}_{s}$ (which is equivalent to the eigenvalue problem since the real part of $\tilde{\mathbf{G}}_{s}$ is symmetric), and will generally be of dimension $n \times(n-2 p+2)$. Thus a reduced dimension sensor vector $\mathbf{f}_{r}$ may be defined such that $\mathbf{f}=\mathbf{T} \mathbf{f}_{r}$, and is chosen by minimizing

$$
J(\mathbf{f})=\mathbf{f}_{r}^{\top}\left[\mathbf{T}^{\top} \hat{\mathbf{K}} \mathbf{T}\right] \mathbf{f}_{r}
$$

subject to

$$
S_{s}(\mathbf{f})=\mathbf{f}^{\top} \mathbf{G}_{s} \mathbf{f}=\mathbf{f}_{r}^{\top}\left[\mathbf{T}^{\top} \mathbf{G}_{s} \mathbf{T}\right] \mathbf{f}_{r} \neq 0 .
$$

This is easily solved by calculating the eigenvalues of $\mathbf{T}^{\top} \tilde{\mathbf{K}} \mathbf{T}$, and taking the eigenvector corresponding to the smallest eigenvalue, consistent with Eq. (23). An alternative is to maximize the sensitivity $S_{s}(\mathbf{f})$ by choosing $\mathbf{f}_{r}$ as the eigenvector corresponding to the largest eigenvalue of $\mathbf{T}^{\top} \mathbf{G}_{s} \mathbf{T}$. 


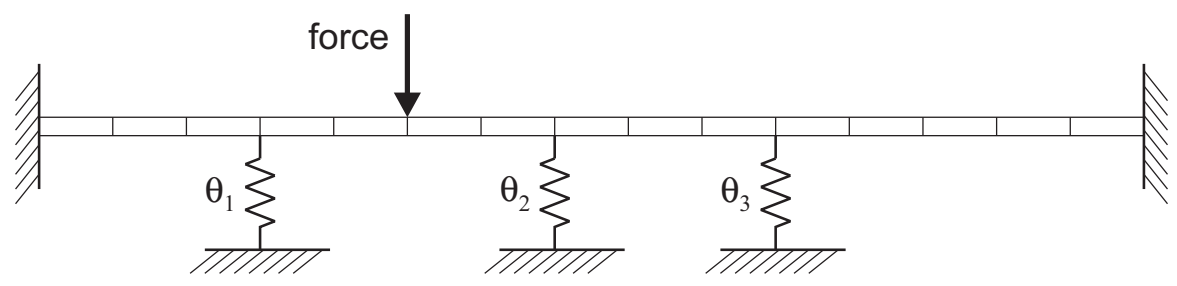

Figure 1. The beam with intermediate supports and harmonic excitation.

\section{A Simulated Beam Example}

A clamped-clamped beam example inspired by Gawronski ${ }^{25}$ will be used here to demonstrate the feasibility of the approach. The steel beam is $1.5 \mathrm{~m}$ long with cross-section $20 \times 5 \mathrm{~mm}$, and bending in the more flexible plane is modeled using 15 finite elements. The beam has three further supports along its length, modeled as springs at nodes 4,8 and 11, where the node numbering convention means that node 1 is clamped, see Fig. 1. The nominal spring stiffnesses of the supports are $10 \mathrm{kN} / \mathrm{m}$. The first four natural frequencies of the system are 32.4, 42.7, 70.1 and $109.0 \mathrm{~Hz}$ and the corresponding modes are shown in Fig. 2. For comparison, the corresponding natural frequencies for the beam without spring supports are 11.8, 32.6, 63.9 and $105.6 \mathrm{~Hz}$. The damping is assumed to be proportional to stiffness with a factor of $10^{-4} \mathrm{~s}$. The force is applied from a rotating machine operating at $40 \mathrm{~Hz}$ at node 6 . The distributed sensor is to be designed for the whole length of the beam initially.

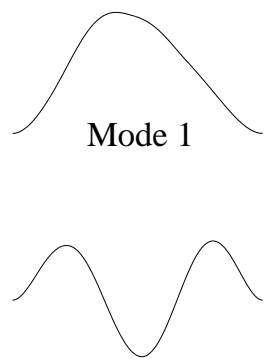

Mode 3
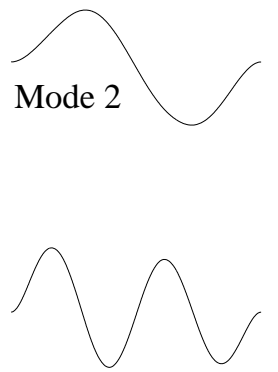

Mode 4

Figure 2. The first four modes of the beam with intermediate supports.

Figure 3(a) shows the sensor shape obtained by minimizing the objective function in Eq. (22) for the first support stiffness. Note that $\mathbf{T}^{\top} \tilde{\mathbf{K}} \mathbf{T}$ has two zero eigenvalues that produce zero sensitivity to the first parameter and are therefore not chosen. The sensor width is normalized so that the maximum width is 1. The result is sensitivity ratios of $S_{1} / S_{2}=1.2 \times 10^{15}$ and $S_{1} / S_{3}=8.7 \times 10^{12}$, demonstrating that the required sensitivity is obtained. Also shown is the frequency response between the force input location and the sensor output. The baseline (solid line) is based on the undamaged system, and the responses due to a $10 \%$ reduction in each of the three support stiffnesses in turn are also shown. Remember that the sensor has been designed to operate only at $40 \mathrm{~Hz}$, and at this frequency the response is indeed insensitive to changes in support stiffnesses 2 and 3. Figure 3(b) shows the equivalent plots when the sensor is designed to be sensitive to support stiffness 2 , also at $40 \mathrm{~Hz}$. It is clear that the procedure has designed sensors with relatively simple shapes.

Suppose now that the sensor shape is chosen to maximize the sensitivity to the first parameter. The result is shown in Fig. 4(a), and highlights that the sensor shape is now more complicated. The sensitivity to parameter 1 is now over 600 times higher than that for the sensor in Fig. 3(a). Figure 4(b) shows the effect of restricting the region where the sensor is placed, by setting the elements of the parameter vector $\mathbf{f}$ corresponding to finite element nodes $1-4$ and $11-16$ to zero. The sensor is designed to ensure zero sensitivity to parameters 2 and 3 and the minimum curvature solution is chosen. There is clearly a compromise between the smaller region for the sensor and the complexity of the shape. 

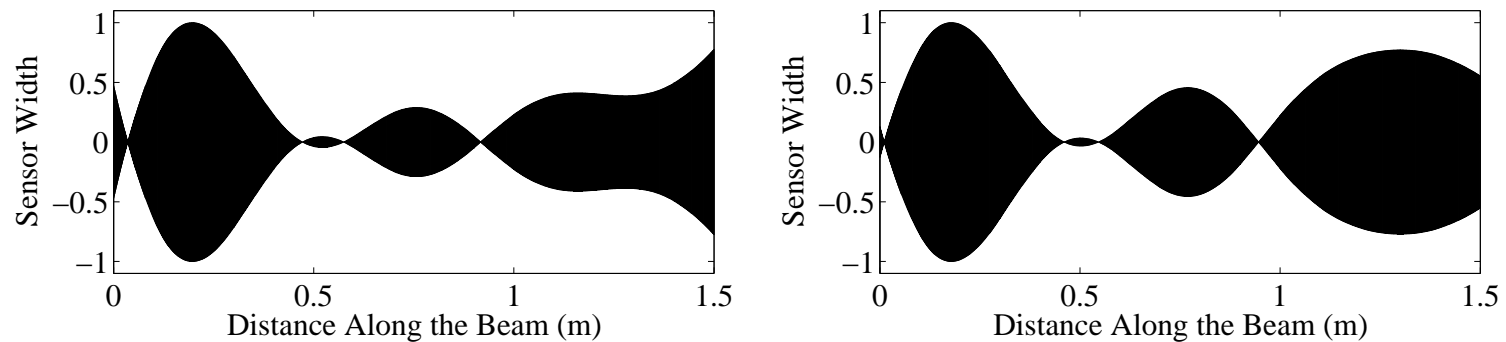

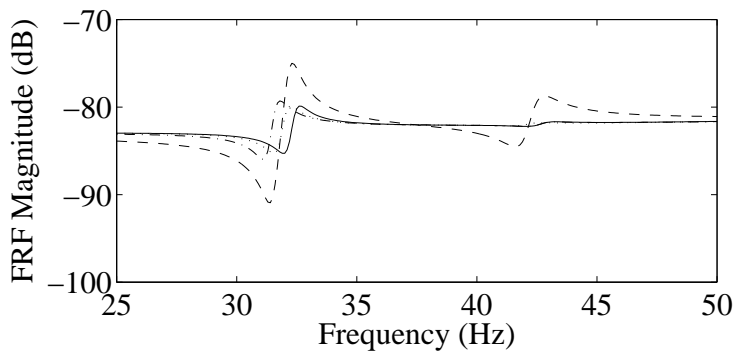

(a) Sensitive to support 1 .

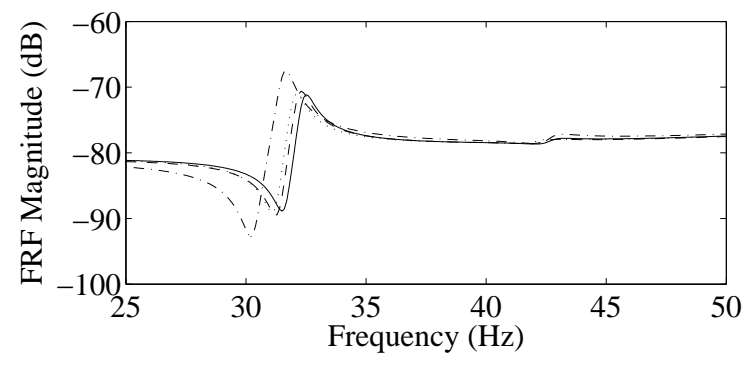

(b) Sensitive to support 2 .

Figure 3. The distributed sensor shape designed to be sensitive to particular support stiffnesses at $40 \mathrm{~Hz}$ for the beam example, and the associated receptances. The solid line is the baseline FRF, the dashed line is due to a $\mathbf{1 0 \%}$ change in support stiffness 1 , the dot-dashed line is due to a change in stiffness 2 , and the dotted line is due to a change in stiffness 3 .

\section{A Simulated Plate Example}

Consider now a rectangular steel plate of dimensions $400 \times 300 \mathrm{~mm}$ and of thickness $7 \mathrm{~mm}$. The plate is supported at each of its corners by a spring of stiffness $500 \mathrm{kN} / \mathrm{m}$. The finite element model has $16 \times 12$ elements, so that each element is $25 \mathrm{~mm}$ square, as shown in Fig. 5. The first four natural frequencies of the system are 21.0, 44.7, 48.8 and $72.7 \mathrm{~Hz}$ and the corresponding modes are shown in Fig. 6. The damping is assumed to be proportional to stiffness with a factor of $10^{-4} \mathrm{~s}$. The force is applied from a rotating machine operating at $40 \mathrm{~Hz}$ at the node shown in Fig. 5 by a star. The distributed sensor is to be designed for the whole plate.

Figure 7 (a) shows the sensor shape sensitive to the first support stiffness, $\theta_{1}$, only and insensitive to the other supports, obtained by minimizing the objective function in Eq. (22). Note that $\mathbf{T}^{\top} \tilde{\mathbf{K}} \mathbf{T}$ has three zero eigenvalues that produce zero sensitivity to the first parameter and are therefore not chosen. The sensor width is normalized so that the maximum thickness is 1 . The result is sensitivity ratios of $S_{1} / S_{2}=7.0 \times 10^{14}, S_{1} / S_{3}=5.6 \times 10^{13}$ and $S_{1} / S_{4}=1.4 \times 10^{12}$, demonstrating that the required sensitivity is obtained. Figure $7(\mathrm{~b})$ shows the sensor shape only sensitive to the second support stiffness, $\theta_{2}$, and the corresponding sensitivity ratios are $S_{2} / S_{1}=1.7 \times 10^{9}, S_{2} / S_{3}=6.3 \times 10^{11}$ and $S_{2} / S_{4}=2.3 \times 10^{9}$. Notice that the shape is very close to that obtained for the first support stiffness and this means that the sensor would have to be manufactured very accurately. Investigations are continuing to determine methods to produce more robust and local sensors for these structures.

\section{Conclusions}

This paper has designed distributed sensors by varying the width (for beams) or thickness (for plates) for structural health monitoring applications. The finite element shape functions are used to define the width or thickness of the sensor and this allows much more flexibility in designing sensors that cover only part of the structure, and other constraints, such as the minimum curvature, may be easily included. It has been demonstrated that sensors may be designed that are sensitive to changes in a single parameter, and insensitive to changes in other parameters. Although much more analysis of these designs is required, 

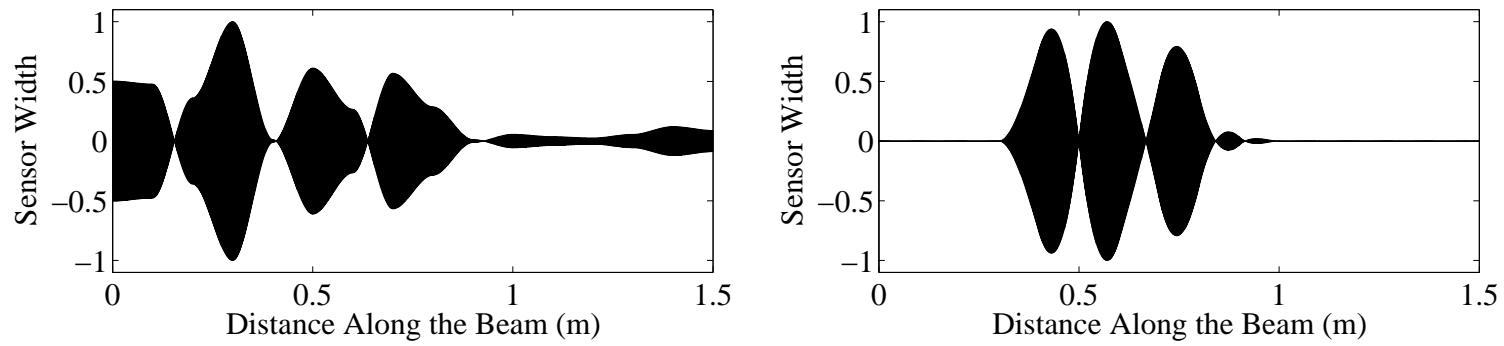

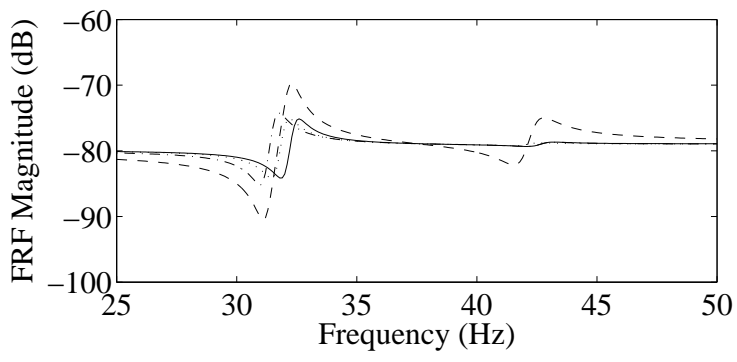

(a) Maximize sensitivity to support 1 .

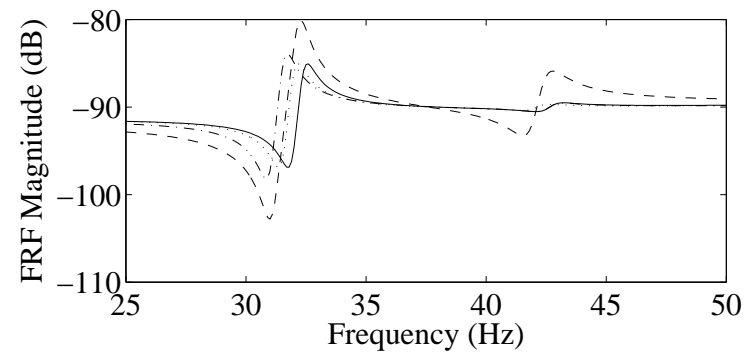

(b) Restrict sensor region.

Figure 4. The distributed sensor shape designed to be sensitive to support stiffness 1 at $40 \mathrm{~Hz}$ for the beam example, and the associated receptances. The solid line is the baseline FRF, the dashed line is due to a $10 \%$ change in support stiffness 1 , the dot-dashed line is due to a change in stiffness 2 , and the dotted line is due to a change in stiffness 3 .

the approach does allow the prospect of a simple distributed sensor to monitor a single region, such as a joint, and be insensitive to other changes, such as those arising from environmental changes. The output from such a sensor could have a threshold alarm system, allowing a robust but simple distributed structural health monitoring system.

\section{Acknowledgments}

SA gratefully acknowledges the support of the Engineering and Physical Sciences Research Council through the award of an Advanced Research Fellowship.

\section{References}

${ }^{1}$ Lee, C.-K. and Moon, F. C., "Modal sensors/actuators," Journal of Applied Mechanics, Vol. 57, 1990, pp. 434-441.

${ }^{2}$ Clark, R. L. and Burke, S. E., "Practical limitations in achieving shaped modal sensors with induced strain materials,"

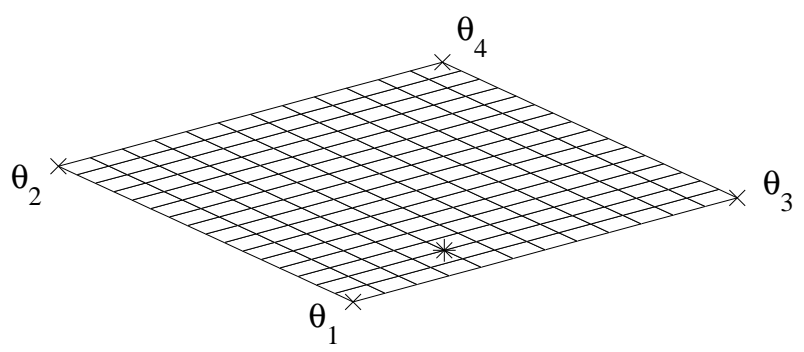

Figure 5. The layout of the plate with corner support springs. The crosses denote the springs and the star denotes the forcing position. 


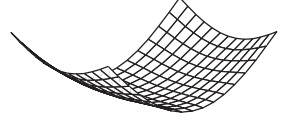

Mode 1

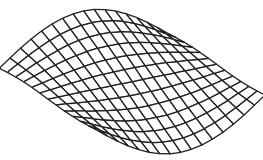

Mode 2

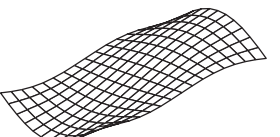

Mode 3

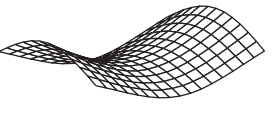

Mode 4

Figure 6. The first four modes of the plate with corner supports.

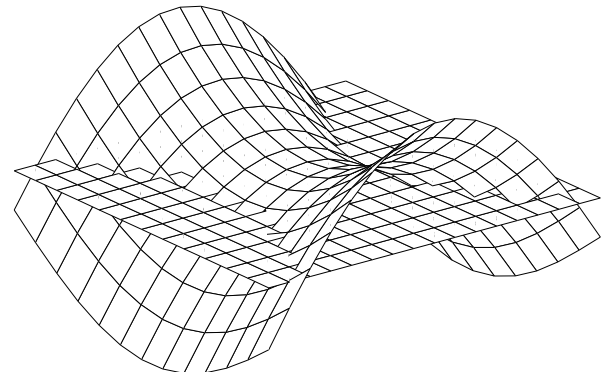

(a) Maximize sensitivity to support 1.

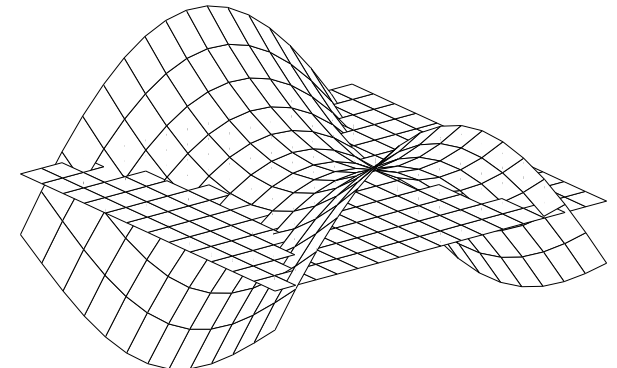

(b) Maximize sensitivity to support 2 .

Figure 7. The distributed sensor shape designed to be sensitive to the support stiffnesses at $40 \mathrm{~Hz}$ for the plate example. 
Journal of Vibration and Acoustics, Vol. 118, 1996, pp. 668-675.

${ }^{3}$ Friswell, M. I., "Partial and segmented modal sensors for beam structures," Journal of Vibration and Control, Vol. 5, 1999, pp. 619-637.

${ }^{4}$ Friswell, M. I., "On the design of modal actuators and sensors," Journal of Sound and Vibration, Vol. 241, 2001, pp. 361-372.

${ }^{5}$ Sun, D., Tong, L., and Wang, D., "Modal actuator/sensor by modulating thickness of piezoelectric layers for smart plates," AIAA Journal, Vol. 40, 2002, pp. 16761679.

${ }^{6}$ Kim, J., Hwang, J.-S., and Kim, S., "Design of modal transducers by optimizing spatial distribution of discrete gain weights," AIAA Journal, Vol. 39, 2001, pp. 19691976

${ }^{7} \mathrm{Kim}$, J., Ryou, J.-K., and Kim, S., "Optimal gain distribution for two-dimensional modal transducer and its implementation using multi-layered PVDF films," Journal of Sound and Vibration, Vol. 251, 2002, pp. 395408.

${ }^{8}$ Preumont, A., Francois, A., Man, P. D., and Piefort, V., "Spatial filters in structural control," Journal of Sound and Vibration, Vol. 265, 2003, pp. 6179.

${ }^{9}$ Jian, K. and Friswell, M. I., "Designing distributed modal sensors for plate structures using finite element analysis," Mechanical Systems and Signal Processing, Vol. 20, 2006, pp. 2290-2304

${ }^{10}$ Jian, K. and Friswell, M. I., "Distributed modal sensors for rectangular plate structures," Journal of Intelligent Material Systems and Structures, Vol. 18, No. 9, 2007, pp. 939-948.

${ }^{11}$ Tanaka, N. and Sanada, T., "Modal control of a rectangular plate using smart sensors and smart actuators," Smart Materials and Structures, Vol. 16, 2007, pp. 36-46.

${ }^{12}$ Doebling, S. W., Farrar, C. R., and Prime, M. B., "A summary review of vibration-based damage identification methods," Shock and Vibration Digest, Vol. 30, 1998, pp. 91105.

${ }^{13}$ Cawley, P. and Adams, R. D., "The locations of defects in structures from measurements of natural frequencies," Journal of Strain Analysis, Vol. 14, 1979, pp. 4957.

${ }^{14}$ Friswell, M. I., Penny, J. E. T., and Wilson, D. A. L., "Using vibration data and statistical measures to locate damage in structures," Modal Analysis: The International Journal of Analytical and Experimental Modal Analysis, Vol. 9, 1994, pp. 239254

${ }^{15}$ Doebling, S. W., Peterson, L. D., and Alvin, K. F., "Estimation of reciprocal residual flexibility from experimental modal data," AIAA Journal, Vol. 34, 1996, pp. 16781685.

${ }^{16}$ Friswell, M. I. and Penny, J. E. T., "Crack modelling for structural health monitoring," Structural Health Monitoring: An International Journal, Vol. 1, 2002, pp. 139-148.

${ }^{17}$ Friswell, M. I., "Damage identification using inverse methods," Royal Society Philosophical Transactions, Vol. 365, 2007, pp. 393-410.

${ }^{18}$ Sohn, H., Worden, K., and Farrar, C. R., "Statistical damage classification under changing environmental and operational conditions," Journal of Intelligent Material Systems and Structures, Vol. 13, 2002, pp. 561-574.

${ }^{19}$ Dawe, D., Matrix and Finite Element Displacement Analysis of Structures, Oxford University Press, 1984.

${ }^{20}$ Park, G., Cudney, H. H., and Inman, D. J., "An integrated health monitoring technique using structural impedance sensors," Journal of Intelligent Material Systems and Structures, Vol. 11, 2000, pp. 448-455.

${ }^{21}$ Ben-Haim, Y., "Adaptive diagnosis of faults in elastic structures by static displacement measurement: the method of selective sensitivity," Mechanical Systems and Signal Processing, Vol. 6, 1992, pp. 85-96.

${ }^{22}$ Cogan, S., Lallement, G., and Ben-Haim, Y., "Updating linear elastic models with modal-based selective sensitivity," 12th International Modal Analysis Conference, 1994.

${ }^{23}$ Ben-Haim, Y. and Prells, U., "Selective sensitivity in the frequency domain, - I. theory," Mechanical Systems and Signal Processing, Vol. 7, 1993, pp. 461-475.

${ }^{24}$ Prells, U. and Ben-Haim, Y., "Selective sensitivity in the frequency domain, - I. applications," Mechanical Systems and Signal Processing, Vol. 7, 1993, pp. 551-574.

${ }^{25}$ Gawronski, W., "Modal actuators and sensors," Journal of Sound and Vibration, Vol. 229, 2000, pp. 1013-1022. 